\title{
Functional brain neuroimaging as a tool to study gastrointestinal manifestations in patients with Parkinson's disease
}

\author{
Ryuji Sakakibara ${ }^{1}$
}

Received: 18 January 2022 / Accepted: 19 January 2022 / Published online: 21 January 2022

(c) The Author(s), under exclusive licence to Springer-Verlag GmbH Germany 2022

Keywords Constipation · Synucleinopathy $\cdot$ Parkinson's disease $\cdot$ Bowel $\cdot$ Autonomic $\cdot$ Insula $\cdot$ Cerebellum $\cdot$ fMRI

Bladder and bowel functions are regulated by complex neuronal networks. The bladder and terminal bowel receive sympathetic (hypogastric nerve), parasympathetic (pelvic nerve), and somatic (pudendal nerve) inputs. Sympathetic innervation of the bowel and bladder originates in the lower thoracic and upper lumbar spinal cord segments and regulates continence of urine and stool, whereas parasympathetic innervation controls the musculature of the bladder and bowel and is responsible for micturition and defecation [1]. The role of the central nervous system in the regulation of bowel and bladder functions remains poorly understood. As a general rule, the brain is thought to have an inhibitory influence on the bladder; suprapontine lesions result in decreased bladder capacity and detrusor overactivity manifesting with predominantly storage symptoms (urinary urgency and/or incontinence). Spinal cord lesions above the lumbosacral level eliminate voluntary and supraspinal control of voiding, leading initially to an areflexic bladder and urinary retention, followed by slow development of automatic micturition and neurogenic detrusor overactivity mediated by spinal reflex pathways. Peripheral nerve lesions are associated with predominantly voiding symptoms with impaired detrusor contractility [1]. As for bowel function, the brain, spinal cord, and peripheral nerves are thought to have a facilitatory effect; lesions cause slower colonic transit time, decreased colonic contraction, anismus, constipation, and paralytic ileus, however, exceptions exist $[1,2]$. The parasympathetic innervation of the gut is provided by the vagus nerve except for the descending colon, sigmoid, and rectum, which are innervated by the sacral parasympathetic

Ryuji Sakakibara

sakakibara@sakura.med.toho-u.ac.jp

1 Neurology, Internal Medicine, Sakura Medical Center, Toho University, 564-1 Shimoshizu, Sakura, Chiba 285-8741, Japan system. Moreover, the enteric nervous system is capable of independent function and intrinsic pacemaker activity from the interstitial cells of Cajal.

The role of supratentorial and brainstem structures in control of lower gastrointestinal function in humans is less well documented than in control of bladder function and data from experimental models and human studies are limited. Functional brain magnetic resonance imaging (fMRI) has led to significant advances in the understanding of human central autonomic network function. Increased activity in different brain regions including the anterior cingulate cortex, insular cortex, prefrontal cortex, and thalamus have been documented using fMRI in patients with functional gastrointestinal disorders (e.g., irritable bowel syndrome with constipation) [3,4]. Constipation is the most frequently reported nonmotor gastrointestinal symptom of Parkinson's disease (PD). James Parkinson himself, in the classic "An Essay on the Shaking Palsy" published in 1817, described severe constipation requiring mechanical removal of the feces [5]. More than a century later, the pathophysiology of constipation in PD remains poorly understood. Several factors including lack of mobility, side effects of medications, and reduced fluid intake may promote constipation in patients with PD. Disease-related pathomechanisms have been identified and evidence suggests that delayed colonic transit in PD stems from disordered central as well as peripheral parasympathetic system dysregulation [6]. The study by Zheng and colleagues is the first to investigate functional changes in the central nervous system associated with bowel dysfunction in patients with PD [7]. The authors studied 106 patients with PD with constipation and 73 patients with PD without constipation. They analyzed resting-state functional connectivity which measures the temporal correlation of spontaneous blood-oxygen-level-dependent (BOLD) signal among spatially distributed brain regions, with the assumption that regions with correlated activity form functional 
networks. In the group of patients with constipation, they found increased low-frequency fluctuation in the right dorsal pons extending into the cerebellum and in the right insula. They also found differences in functional connections linking the superior temporal gyrus, particularly the right superior temporal gyrus, with multiple brain regions [7].

Of particular interest, the dorsal pons, cerebellum, and right insula are important structures for the control of bladder function. Among these brain areas, the dorsal pons includes the pontine defecation center (that is identical to the pontine micturition center located in the locus coeruleus). Increased activity in the insula has been reported in patients with irritable bowel syndrome with constipation [8], while the role of the cerebellum in the regulation of bowel function is not well understood [9]. The significance of the increased connectivity between the right superior temporal gyrus (exact location not shown) and other brain regions in patients with constipation is a matter of debate. Although the prefrontal cortex, cingulate cortex, and insular cortex are involved in the control of bowel function, the role of the temporal cortex has not been delineated [10]. In summary, the study by Zheng and colleagues is promising and paves the way for future research exploring the neural substrate of bowel dysfunction in PD. It would also be interesting to evaluate fMRI changes after pharmacological and nonpharmacological interventions for constipation in PD.

\section{Funding None.}

\section{Declarations}

Conflict of interest The authors declare that they have no conflict of interest.

\section{References}

1. Drake MJ, Fowler CJ, Griffiths D, Mayer E, Paton JF, Birder L (2010) Neural control of the lower urinary and gastrointestinal tracts: supraspinal CNS mechanisms. Neurourol Urodyn 29(1):119-127

2. Ito T, Sakakibara R, Uchiyama T, Zhi L, Yamamoto T, Hattori $\mathrm{T}$ (2006) Videomanometry of the pelvic organs: a comparison of the normal lower urinary and gastrointestinal tracts. Int J Urol 13(1):29-35

3. Kano M, Grinsvall C, Ran Q, Dupont P, Morishita J, Muratsubaki T et al (2020) Resting state functional connectivity of the pain matrix and default mode network in irritable bowel syndrome: a graph theoretical analysis. Sci Rep 10(1):11015

4. Kano M, Muratsubaki T, Yagihashi M, Morishita J, Mugikura S, Dupont P et al (2020) Insula activity to visceral stimulation and endocrine stress responses as associated with alexithymia in patients with irritable bowel syndrome. Psychosom Med 82(1):29-38

5. Parkinson J (1817) An essay on the shaking palsy. Whittingham \& Rowland, London

6. Borghammer P (2018) Is constipation in Parkinson's disease caused by gut or brain pathology? Parkinsonism Relat Disord 55:6-7

7. Zheng JH, Sun WH, Ma JJ, Wang ZD, Chang QQ, Dong LR et al (2022) Resting-state functional magnetic resonance imaging in patients with Parkinson's disease with and without constipation: a prospective study. Clin Auton Res (ahead of print)

8. Nakamori H, Naitou K, Horii Y, Shimaoka H, Horii K, Sakai $\mathrm{H}$ et al (2019) Roles of the noradrenergic nucleus locus coeruleus and dopaminergic nucleus A11 region as supraspinal defecation centers in rats. Am J Physiol Gastrointest Liver Physiol 317(4):G545-G555

9. Sakakibara R (2019) The cerebellum seems not a 'little brain' for the autonomic nervous system. Clin Neurophysiol 130(1):160

10. Tateno F, Sakakibara R, Yano M, Takahashi O, Sugiyama M, Kishi M et al (2014) A young man with herpes simplex encephalitis: Andrew and Nathan type urodynamic abnormality. Bladder $1: 4$ 